\title{
The Jones polynomial for fluid knots from helicity
}

\author{
Xin Liu ${ }^{1}$ and Renzo L Ricca ${ }^{2,3}$ \\ ${ }^{1}$ School of Mathematics and Statistics, University of Sydney, NSW 2006, Australia \\ 2 Department of Mathematics and Applications, University of Milano-Bicocca, Via Cozzi 53, \\ 20125 Milano, Italy \\ E-mail: liuxin@maths.usyd.edu.au and renzo.ricca@unimib.it
}

Received 15 December 2011

Published 4 May 2012

Online at stacks.iop.org/JPhysA/45/205501

\begin{abstract}
In this paper we prove that under ideal conditions the helicity of fluid knots, such as vortex filaments or magnetic flux tubes, provides a fundamentally new topological means by which we may associate a topological invariant, the Jones polynomial, that is much stronger than prior interpretations in terms of Gauss linking numbers. Our proof is based on an extension of the Kauffman bracket polynomial for unoriented knots. Explicit calculations of the Jones polynomial for the left- and right-handed trefoil knots and for the Whitehead link via the figure-of-eight knot are presented for illustration. This novel approach establishes a topological foundation of classical field theory in general, and of mathematical fluid dynamics in particular, by opening up new directions of work both in theory and applications.
\end{abstract}

PACS numbers: 11.10.-z, 47.10.A, 47.32.C-, 52.30.Cv

(Some figures may appear in colour only in the online journal)

\section{Introduction}

In this paper we prove that in ideal conditions the helicity of fluid knots, such as vortex filaments or magnetic flux tubes, provides a fundamentally new topological means by which we may associate a topological invariant, the Jones polynomial, that is much stronger than prior interpretations in terms of Gauss linking numbers. This result provides a fundamentally new topological approach to the study of complex fluid flows, by extending the former use of helicity in terms of linking numbers to the computation of knot polynomials. This novel approach contributes to establish a topological foundation of classical field theory in general, and of mathematical fluid dynamics in particular, by opening up new directions of work both in theory and applications.

3 Author to whom any correspondence should be addressed. 
It is well known that helicity plays a fundamental role both in fluid mechanics and magnetohydrodynamics (MHD), it being an invariant of the Euler and ideal MHD equations, and a robust quantity of the dissipative Navier-Stokes equations and resistive MHD flows. In ideal conditions its invariance was first established by Woltjer (1958) in the context of MHD (magnetic helicity), and its topological interpretation in terms of the Gauss linking number was provided by Moffatt (1969) in the context of vortex dynamics (kinetic helicity). In the latter case, kinetic helicity is defined by

$$
H \equiv \int_{\Omega} \mathbf{u} \cdot \omega \mathrm{d}^{3} \mathbf{x}
$$

where $\mathbf{u}$ is the velocity field, defined on an unbounded domain of $\mathbb{R}^{3}, \omega=\nabla \times \mathbf{u}$ is the vorticity, defined on a sub-domain $\Omega$ and $\mathbf{x}$ is a position vector in $\mathbb{R}^{3}$. For simplicity, we assume $\nabla \cdot \mathbf{u}=0$ everywhere and request $\boldsymbol{\omega} \cdot \hat{\mathbf{n}}=0$ on $\partial \Omega$, where $\hat{\mathbf{n}}$ is orthogonal to $\partial \Omega$, with $\nabla \cdot \omega=0$. Magnetic helicity, on the other hand, is simply obtained by replacing $\mathbf{u} \cdot \boldsymbol{\omega}$ with $\mathbf{A} \cdot \mathbf{B}$, where $\mathbf{B}=\nabla \times \mathbf{A}$ is the magnetic field in $\Omega$ and $\mathbf{A}$ is its vector potential, subject to the Coulomb gauge condition $\nabla \cdot \mathbf{A}=0$. Indeed, any form of the type

$$
H=\int_{M^{3}} A \wedge \mathrm{d} A
$$

is a helicity-type quantity, where $M^{3}$ is a compact three-manifold with coordinates $x^{i}$ ( $i=1,2,3)$, and the gauge potential $A=A_{i}^{a} \tau_{a} \mathrm{~d} x^{i}$ is a connection 1-form with $\tau_{a}$ ( $a=1, \ldots, \operatorname{dim}(G)$ ) being the generators of the gauge group $G$. It was Whitehead (1947), who showed that indeed $H$ is an isotopy invariant of $M^{3}$.

The topological meaning of helicity is particularly evident when we consider vortex filaments in $\mathbb{R}^{3}$. For $N$ thin vortex filaments, $\Omega=\sum_{i} \Omega_{i}(i=1, \ldots, N)$ is the union of $N$ tubular regions $\Omega_{i}$, with vorticity embedded in $\Omega_{i}$. Each vortex is centred on a smooth, possibly knotted, oriented curve $\gamma_{i}$, central axis of $\Omega_{i}$. A collection of knots forms a link, and in general we shall refer to a collection of vortex filaments as an oriented knot or link $\mathcal{K}$ in $\mathbb{R}^{3}$. Thus, the helicity $H=H(\mathcal{K})$ is given by (Ricca and Moffatt 1992, Moffatt and Ricca 1992)

$$
H(\mathcal{K})=\sum_{i} \kappa_{i}^{2} L k_{i}+2 \sum_{i j} \kappa_{i} \kappa_{j} L k_{i j}
$$

where $\kappa_{i}$ denotes vortex circulation, $L k_{i}$ denotes the (Călugăreanu 1961, White 1969) selflinking number of vorticity lines in $\Omega_{i}$ with respect to the centerline $\gamma_{i}$, and $L k_{i j}$ is the Gauss linking number of $\gamma_{i}$ with $\boldsymbol{\gamma}_{j}$. Note that the self-linking number of the $i$ th-vortex admits decomposition in terms of the writhing number $\left(W r_{i}\right)$ and twist number $\left(T w_{i}\right)$, according to $L k_{i}=W r_{i}+T w_{i}$, two quantities that account for the geometry of the $i$ th vortex filament in space. The circulation (or topological charge) $\kappa_{i}$ is an invariant of the Euler equations and provides information on the topological complexity (connectedness) of the fluid domain in $\mathbb{R}^{3}$.

As Maxwell pointed out (1873, vol II, section 4.1; see also Ricca and Nipoti 2011 for a historical reconstruction), it is known that the linking number sometimes fails to detect essential topology, as the examples of the Whitehead link and the Borromean rings demonstrate. A richer topological description is therefore needed to extend the use of helicity beyond linking numbers. A possibility is provided by the computation of polynomial knot invariants. These provide a tool by which one can define stronger means to distinguish families of knots and links. Kauffman's bracket polynomial definition of the Jones polynomial suggests this as an attractive first step, even though stronger methods, such as the Khovanov homology or the Heegaard Floer homologies, are known to be significantly more successful. Our application of the Kauffman method will be accomplished by an approach similar to that done in topological 
quantum field theory (TQFT). By recalling some basic results of TQFT, we shall first reinterpret helicity as an Abelian Chern-Simons (CS) action (section 2). In section 3 we shall recall some basic concepts of knot theory by introducing the $R$-polynomial for oriented knots, that will serve as a preliminary tool for the subsequent derivation. Our main result, i.e. a description of a method by which the Jones polynomial can be used to study the topology of fluid knots and links (vortex or magnetic) through helicity, will be stated and demonstrated in section 4. This will be done in two steps: first, by using the Kauffman bracket polynomial for unoriented knots (section 4.1) we shall derive the three skein relations, and then, by recovering orientation, we shall re-write the skein relations of the Jones polynomial (section 4.2). The cases of left- and right-handed trefoil knots (mirror knots), and the case of the Whitehead link via the figure-of-eight knot, will be discussed in section 5 as explicit examples of polynomial calculations for simple knots and links. Conclusions will be drawn in section 6 .

\section{Helicity as an Abelian Chern-Simons action}

In gauge field theory the non-Abelian Chern-Simons (CS) action is given by

$$
S=\int_{M^{3}} A \wedge \mathrm{d} A+A \wedge A \wedge A .
$$

When the gauge group $G$ is Abelian, then equation (4) reduces to $S_{\text {Abelian }}=\int_{M^{3}} A \wedge \mathrm{d} A$ (cf equation (2) above). Thus, the helicity $H$ is an Abelian CS action.

Quantum CS field theory (see, for example, Peskin and Schoeder 1995) studies the vacuum expectation value of the Wilson loops, essentially given by

$$
\left\langle\prod_{k=1}^{N} \mathrm{e}^{\mathrm{i} \oint_{\gamma_{k}} A}\right\rangle=\frac{1}{Z} \int[\mathcal{D} A] \mathrm{e}^{\sum_{k} \mathrm{i} \oint_{\gamma_{k}} A} \mathrm{e}^{\mathrm{i} S},
$$

where $\gamma_{k}$ is the (unoriented) support of a Wilson loop and $Z=\int[\mathcal{D} A] \mathrm{e}^{\mathrm{i} S}$ is the partition function. Work done in this context, initiated by Witten (1989) and carried out by many others (see, for example, Le et al 1998), shows that the functional integral in (5) admits a topological interpretation in terms of the Jones (1987), HOMFLY-PT (Freyd et al 1985, Przytycki and Traczyk 1987) and other knot polynomials (Kauffman 1987, 1991). Moreover, from the exponential expansion of (5) we can extract the Vassiliev invariants of knots and three-manifolds via the Kontsevich integrals (see, for example, Altschuler and Freidel 1997, Chmutov et al 2011), and the linking numbers of knots (see, for instance, Bar-Natan 1995, Labastida and Pérez 1998). It is therefore natural to expect that helicity, as an Abelian CS action, gives rise to knot polynomials. This will indeed be the case, and it will be proven in section 4.2 .

Before proceeding further, we need to make one more observation. In the case of thin vortex filaments (and similarly for thin magnetic flux tubes), the vorticity field can be thought of as given by $\boldsymbol{\omega}=\omega_{0} \hat{\mathbf{t}}$, where $\omega_{0}$ is a constant and $\hat{\mathbf{t}}$ is the unit tangent to the vortex axis, so that equation (1) can be reduced (see, for instance, Barenghi et al 2001) to

$$
H=\sum_{k} \kappa_{k} \oint_{\gamma_{k}} \mathbf{u} \cdot \mathrm{d} \mathbf{l},
$$

where $\kappa_{k}=\int_{\Omega_{0 k}} \omega_{0} \mathrm{~d}^{2} \mathbf{x}$ is the circulation, $\Omega_{0 k}$ is the vortex cross-section, $\mathbf{u}$ is the vortex self-induced velocity given by the Biot-Savart integral and dl is an elementary arc-length of $\boldsymbol{\gamma}_{k}$ in the $\hat{\mathbf{t}}$-direction. For simplicity, we can set $\kappa_{k}=1$. Hence, in analogy with equation (5), and up to a constant, we can introduce the functional

$$
\left\langle\prod_{k=1}^{N} \mathrm{e}^{\mathrm{i} \oint_{\gamma_{k}} \mathbf{u} \cdot \mathrm{dl} \mathbf{l}}\right\rangle=\int[\mathcal{D} \mathbf{u}] \mathrm{e}^{\sum_{k} \mathrm{i} \oint_{\gamma_{k}} \mathbf{u} \cdot \mathrm{dl}} \mathrm{e}^{\mathrm{i} H} .
$$




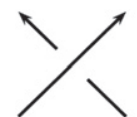

(a)

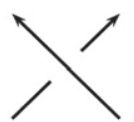

(b)

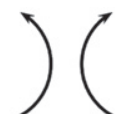

(c)

Figure 1. (a) Over-crossing $\boldsymbol{L}_{+},(b)$ under-crossing $\boldsymbol{L}_{-}$and (c) non-crossing $\boldsymbol{L}_{0}$ of oriented strands in a knot diagram.

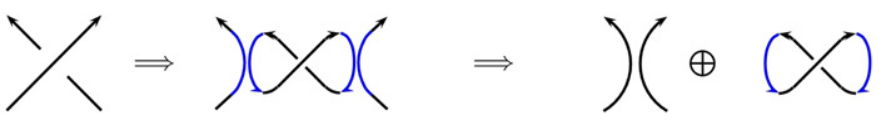

(a)

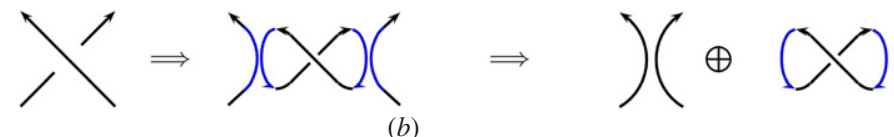

Figure 2. By adding and subtracting local paths $(a)$ an over-crossing is reduced to a non-crossing plus a positive writhe $\boldsymbol{\gamma}_{+}$and $(b)$ an under-crossing is reduced to a non-crossing plus a negative writhe $\boldsymbol{\gamma}_{-}$.

Note that now the (oriented) integration paths coincide with the vortex centerlines $\boldsymbol{\gamma}_{k}$. Thus, by using equation (6) above, we have

$$
\left\langle\prod_{k=1}^{N} \mathrm{e}^{\mathrm{i} \oint_{\gamma_{k}} \mathbf{u} \cdot \mathrm{dl}}\right\rangle=\int[\mathcal{D} \mathbf{u}]\left(\mathrm{e}^{\sum_{k} \mathrm{i} \oint_{\gamma_{k}} \mathbf{u} \cdot \mathrm{d} \mathbf{l}}\right)^{2}=\int[\mathcal{D} \mathbf{u}]\left(\mathrm{e}^{\mathrm{i} H}\right)^{2} .
$$

From the considerations made above, and from direct inspection of the right-hand side of equation (8), it is evident that by straightforward re-scaling we can replace $\mathrm{e}^{\mathrm{i} H}$ with $t^{H}$ (the base e being just a constant). Hence, by following a strategy similar to that outlined by Birman and Lin (1993), we can re-define $t^{H}$ as a new variable and use this new variable to show that indeed $t^{H}$ satisfies the skein relations of knot polynomials.

\section{3. $R$-polynomial for oriented knots}

We need to recall some preliminary information on the $R$-polynomial for oriented knots. Kauffman (1987) proved that the Jones knot polynomial can be constructed by analyzing crossing states in a knot diagram, where (see figure 1) over-crossings, under-crossings and non-crossings are denoted by $\boldsymbol{L}_{+}, \boldsymbol{L}_{-}$and $\boldsymbol{L}_{0}$, respectively.

Calculations are based on applying reduction techniques performed recursively on crossing sites, according to the diagrams shown in figure 2 . These techniques resort to virtually split the over/under-crossing (figures $2(a)$ and $(b)$ ), by adding and subtracting local paths, so as to reduce each crossing site to a non-crossing plus a positive/negative writhe contribution, denoted respectively by $\boldsymbol{\gamma}_{+}$and $\boldsymbol{\gamma}_{-}$. These operations can be symbolically represented by a single equation in the variable $t^{H}$, given by

$$
t^{H\left(\boldsymbol{L}_{ \pm}\right)}=t^{H\left(\boldsymbol{L}_{0} \oplus \boldsymbol{\gamma}_{ \pm}\right)}=t^{H\left(\boldsymbol{L}_{0}\right)} t^{H\left(\boldsymbol{\gamma}_{ \pm}\right)} .
$$

By definition, $H\left(\boldsymbol{\gamma}_{ \pm}\right)=\oint_{\gamma_{ \pm}} \mathbf{u} \cdot \mathrm{d} \mathbf{l}$ is given by the self-linking number associated with $\boldsymbol{\gamma}_{ \pm}$; hence, we can set $H\left(\boldsymbol{\gamma}_{ \pm}\right)= \pm 1 / 2$ (or any other constant value between 0 and \pm 1 ). Thus, 


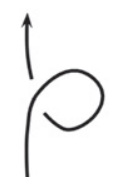

(a)

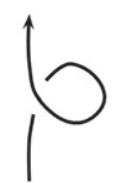

(b)

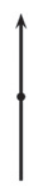

(c)

Figure 3. ( $a$ ) Writhe +1 , denoted by $\boldsymbol{\alpha}_{+},(b)$ writhe -1 , denoted by $\boldsymbol{\alpha}_{-}$and $(c)$ non-writhe, denoted by $\boldsymbol{\alpha}_{0}$.

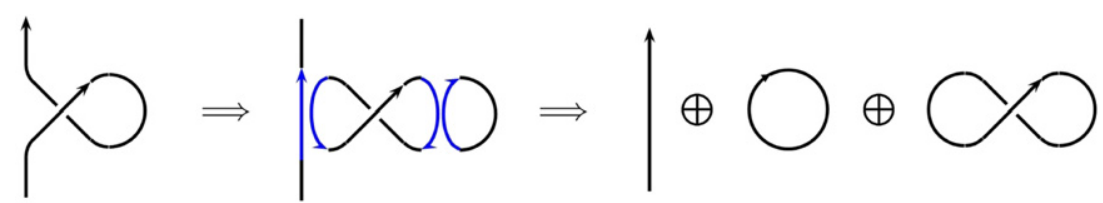

Figure 4. By adding and subtracting local paths, a writhe +1 is reduced to a trivial circle plus a writhe $\boldsymbol{\gamma}_{+}$.

by taking the difference between the two states and by setting $k=t^{1 / 2}$, we have a first skein relation for the knot polynomial, that is,

$$
t^{H\left(\boldsymbol{L}_{+}\right)}-t^{H\left(\boldsymbol{L}_{-}\right)}=\left(k-k^{-1}\right) t^{H\left(\boldsymbol{L}_{0}\right)} .
$$

The second skein relation is obtained by considering the writhe states $\boldsymbol{\alpha}_{+}, \boldsymbol{\alpha}_{-}$and $\boldsymbol{\alpha}_{0}$ as shown in figure 3. As above, by virtually adding and subtracting local paths to each writhe state, as shown by the example of figure 4 , we can obtain relationships between $\boldsymbol{\alpha}_{ \pm}$and $\boldsymbol{\alpha}_{0}$.

Assuming that the self-linking number of a trivial circle is zero, then $t^{H(\bigcirc)}=1$, and so we obtain

$$
t^{H\left(\boldsymbol{\alpha}_{+}\right)}=t^{H\left(\boldsymbol{\alpha}_{0}\right)} t^{H\left(\boldsymbol{\gamma}_{+}\right)}=k t^{H\left(\boldsymbol{\alpha}_{0}\right)} .
$$

Similarly for $\boldsymbol{\alpha}_{-}$:

$$
t^{H\left(\boldsymbol{\alpha}_{-}\right)}=k^{-1} t^{H\left(\boldsymbol{\alpha}_{0}\right)} .
$$

Thus, by defining $R(\mathcal{K})=t^{H(\mathcal{K})}$ for an oriented knot (or link) $\mathcal{K}$, and by setting $z=k-k^{-1}$, equations (10)-(12) are re-written as

$$
\begin{aligned}
& R(\bigcirc)=1, \\
& R\left(\boldsymbol{\alpha}_{+}\right)=k R\left(\boldsymbol{\alpha}_{0}\right), \quad R\left(\boldsymbol{\alpha}_{-}\right)=k^{-1} R\left(\boldsymbol{\alpha}_{0}\right), \\
& R\left(\boldsymbol{L}_{+}\right)-R\left(\boldsymbol{L}_{-}\right)=z R\left(\boldsymbol{L}_{0}\right) .
\end{aligned}
$$

Equations (13)-(15) are the skein relations of the so-called $R$-polynomial originally introduced by Kauffman (1987).

Note that in Kauffman's original notation one has $\delta=\left(k-k^{-1}\right) / z$, whereas in our case we simply set $\delta=1$. While this position is useful for the purpose we have in mind, this choice is inevitably more restrictive, since it leads to a reduction from a two-parameter polynomial to a single-parameter polynomial (from $\delta=1$, we have $z=k-k^{-1}$ ); consequently, the resulting degenerate $R$-polynomial is considerably weaker, failing to distinguish, for example, simple, distinct topologies as shown in figure 5. Therefore, we need to resort to a more powerful knot polynomial, as will be shown in the next section. 


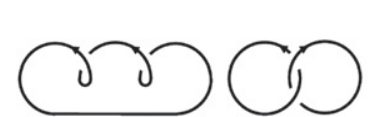

(a)

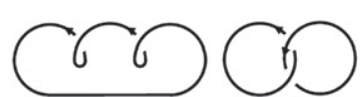

(b)

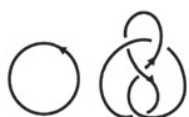

(c)

Figure 5. The $R$-polynomial cannot distinguish between the different topologies in the pairs of knots shown in the three cases above.

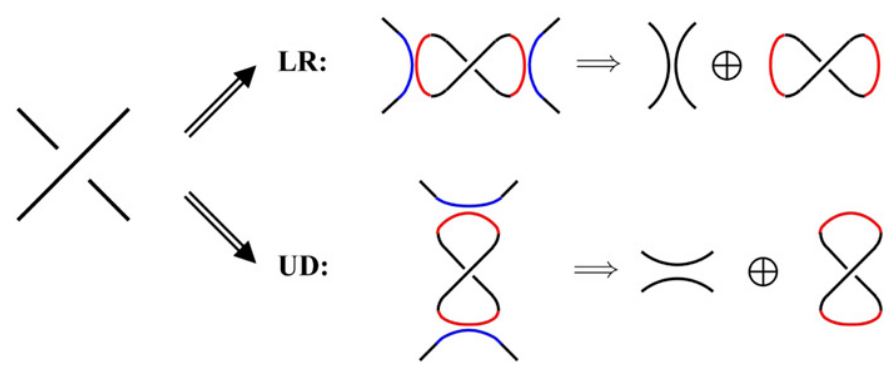

Figure 6. The crossing $L_{+}$can be split by adding and subtracting local paths following the left-right (LR) or up-down (UD) scheme above. Both decompositions contribute equally to the calculation of $t^{H}$.

\section{Jones' polynomial from helicity}

In this section we shall prove that the Jones polynomial of fluid knots, such as vortex or magnetic knots, can be expressed in terms of helicity. The following result holds true.

Theorem 1. Let $\mathcal{K}$ denote a fluid knot or an n-component link. If the helicity of $\mathcal{K}$ is $H=H(\mathcal{K})$, then

$$
t^{H(\mathcal{K})}=t^{\oint_{\mathcal{K}} \mathbf{u} \cdot \mathrm{d} \mathbf{l}},
$$

appropriately rescaled, satisfies the skein relations of the Jones polynomial $V=V(\mathcal{K})$.

Proof of this result is based on a two-step approach. First (part 1, section 4.1), we shall consider unoriented knots and derive the Kauffman bracket polynomial for these knots. Then (part 2, section 4.2), by taking into account orientation, we shall derive the skein relations of the Jones polynomial for oriented knots from the bracket polynomial, and this will complete the proof of the theorem.

\subsection{Proof of theorem 1: Kauffman's bracket polynomial for unoriented knots (part 1)}

Let $\mathcal{K}$ denote an unoriented fluid knot or an $n$-component link. Let us consider the decomposition of the unoriented crossing $L_{+}$of figure 6 . The crossing can be virtually split by adding and subtracting local paths (of opposite 'charge') following the left-right (LR) or up-down (UD) scheme shown in the figure. Evidently, both decompositions have the same probability to occur; therefore, we assume that both must contribute equally to the calculation of $t^{H}$. Hence,

$$
t^{H\left(L_{+}\right)}=t_{\mathrm{LR}}^{H\left(L_{+}\right)}+t_{\mathrm{UD}}^{H\left(L_{+}\right)}
$$

where the terms on the right-hand side refer to the decomposition scheme shown in the figure. Let us examine the contributions from parallel strands and from the figure-of-eight unknot, 

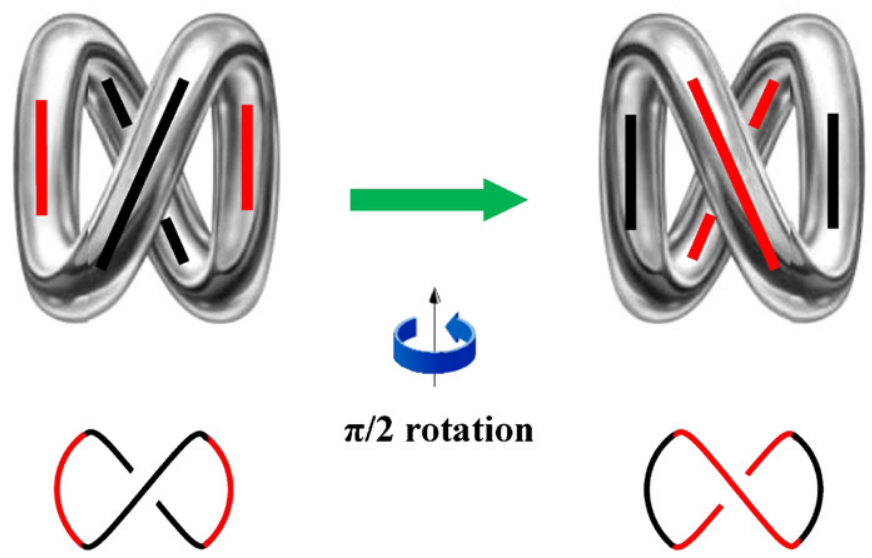

$\pi / 2$ rotation

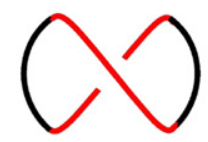

Figure 7. The writhe (absolute) value of a figure-of-eight unknot is bounded between 0 and 1 .

separately. The parallel strands ( do not add any topological information, and indeed they correspond to a non-crossing $L_{0}$. Thus, their contribution to $t^{H}$ is zero.

The writhe contribution of the figure-of-eight unknot $\gamma_{ \pm}$is bounded between 0 and 1 (in absolute value), and it cannot be determined a priori. Therefore, with reference to the LR scheme of figure 6 , the contribution of $\gamma_{+}$to the helicity by its self-linking number is given by $\lambda H\left(\gamma_{+}\right)$, where $0 \leqslant \lambda \leqslant 1$ takes account of the uncertainty associated with the writhe value (see figure 7). Hence, according to the LR decomposition, the unoriented $L_{+}$splits into $L_{0} \oplus \gamma_{+}$, by contributing $t_{\mathrm{LR}}^{H\left(L_{+}\right)}=t^{H\left(L_{0}\right)} t^{\lambda H\left(\gamma_{+}\right)}$to helicity. By taking $a=t^{\lambda H\left(\gamma_{+}\right)}$, we have

$$
t_{\mathrm{LR}}^{H\left(L_{+}\right)}=a t^{H\left(L_{0}\right)} .
$$

Similarly for the UD decomposition, where we have $L_{\infty} \oplus \gamma_{-}$, where $L_{\infty}=\asymp$; thus

$$
H\left(\gamma_{-}\right)=\lambda H\left(\gamma_{-}\right)=-\lambda H\left(\gamma_{+}\right)
$$

Hence

$$
t_{\mathrm{UD}}^{H\left(L_{+}\right)}=a^{-1} t^{H\left(L_{\infty}\right)}
$$

where $a^{-1}=t^{\lambda H\left(\gamma_{-}\right)}=t^{-\lambda H\left(\gamma_{+}\right)}$. By combining equations (17), (18) and (20), we have

$$
t^{H\left(L_{+}\right)}=a t^{H\left(L_{0}\right)}+a^{-1} t^{H\left(L_{\infty}\right)}
$$

The same reasoning can be repeated for the LR and UD decompositions performed on the other crossing $L_{-}$. We have

$$
t^{H\left(L_{-}\right)}=a t^{H\left(L_{\infty}\right)}+a^{-1} t^{H\left(L_{0}\right)}
$$

Evidently, equations (21) and (22) are complementary, and both of them must contribute to the skein relations of the Kauffman bracket polynomial for unoriented knots.

Another skein relation comes from considerations of the contribution from distinct components of a link, that is from $\mid \sqcup \bigcirc$, where $\bigcirc$ denotes a trivial circle and the rest of the link. With reference to figure 8, we see how loop types $\alpha_{+}$and $\alpha_{-}$can be virtually decomposed into a collection of elementary paths by local splitting of strands. From this decomposition we derive the diagram of figure 9 that shows that $\mid \sqcup \bigcirc$ can be obtained by a combination of two LR splits performed on the loop types $\alpha_{+}$and $\alpha_{-}$, i.e.

$$
t^{H\left(\alpha_{+} \ominus \gamma_{+}\right)}=t^{H\left(\alpha_{+}\right)} t^{-H\left(\gamma_{+}\right)}=a^{-1} t^{H\left(\alpha_{+}\right)},
$$



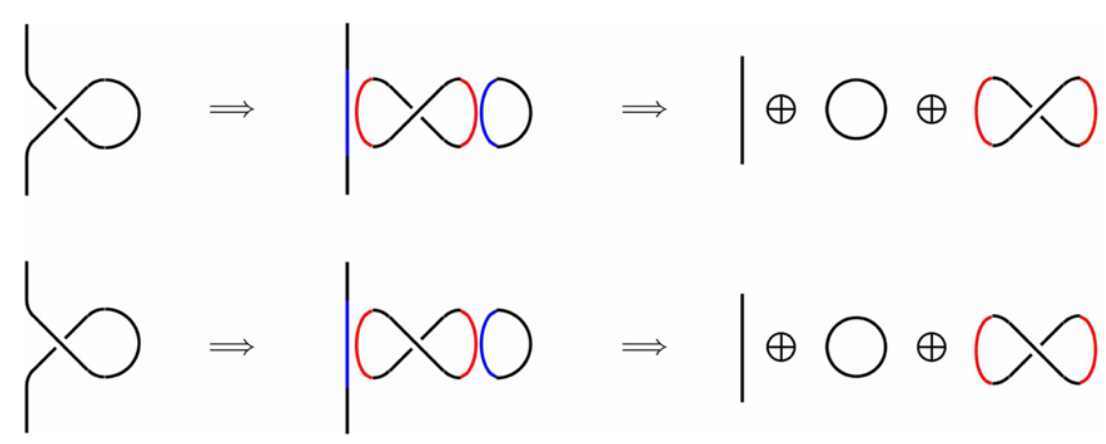

Figure 8. Virtual decomposition of loop types $\alpha_{+}$and $\alpha_{-}$by strand splitting into local paths.

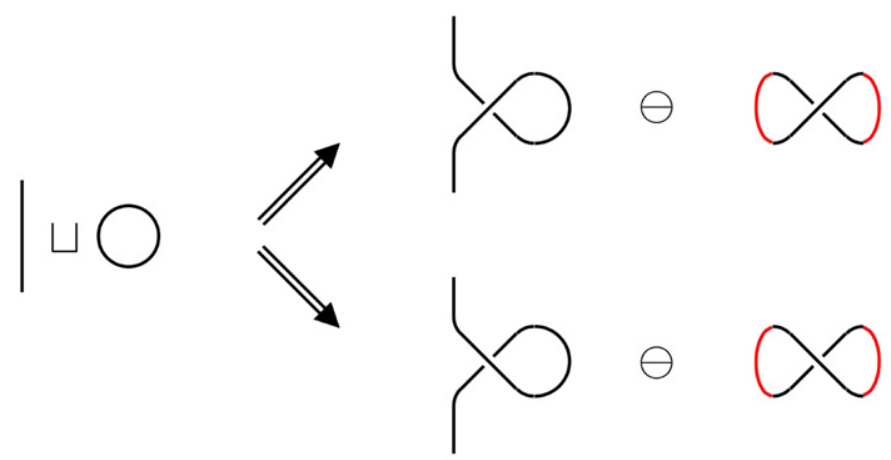

Figure 9. Disjoint union of link components from decomposition of loop types.

and

$$
t^{H\left(\alpha_{-} \ominus \gamma_{-}\right)}=t^{H\left(\alpha_{-}\right)} t^{-H\left(\gamma_{-}\right)}=a t^{H\left(\alpha_{-}\right)} .
$$

Since the two split paths have equal probability of occurring, they must contribute equally to the decomposition $\mid \sqcup \bigcirc$; hence,

$$
t^{\left.H(\mid \sqcup)^{\prime}\right)}=a^{-1} t^{H\left(\alpha_{+}\right)}+a t^{H\left(\alpha_{-}\right)} .
$$

Since the writhes $\alpha_{+}$and $\alpha_{-}$present apparent crossings, and these are described by the skein relations (21) and (22), then we can apply (21) and (22) to $\alpha_{+}$and $\alpha_{-}$to further decompose the right-hand side of the diagram of figure 9 . By a straightforward application of the equations above to $\alpha_{+}$and $\alpha_{-}$, we obtain

$$
\left.t^{H\left(\alpha_{+}\right)}=a t^{H(\mid \sqcup \bigcirc)}+a^{-1} t^{H(} \mid\right),
$$

and

$$
\left.t^{H\left(\alpha_{-}\right)}=a t^{H(} \mid\right)+a^{-1} t^{H(\mid \sqcup \bigcirc) .}
$$

By combining (25) with (26) and (27), we obtain

$$
\left.t^{H(\mid \sqcup \bigcirc)}=-\left(a^{2}+a^{-2}\right) t^{H(} \mid\right),
$$

which provides the last skein relation of the bracket polynomial. 
By introducing the bracket symbol $\langle\mathcal{K}\rangle=t^{H(\mathcal{K})}$ for unoriented knots, we can rewrite (21), (22) and (28) as the skein relations of the Kauffman bracket polynomial, that is,

$$
\begin{aligned}
& \langle\bigcirc\rangle=1, \\
& \left.\langle y\rangle=a\langle)(\rangle+a^{-1}\langle\longleftarrow\rangle, \quad\langle\rangle\right\rangle=a^{-1}\langle)(\rangle+a\langle\approx\rangle, \\
& \langle\mid \sqcup \bigcirc\rangle=-\left(a^{2}+a^{-2}\right)\langle\mid\rangle .
\end{aligned}
$$

\subsection{Proof of theorem 1: Jones' polynomial for oriented knots (part 2)}

The Jones polynomial, an ambient isotopy invariant for oriented knots, is obtained straightforwardly from the Kauffman bracket polynomial, that is, a regular isotopy invariant for unoriented knots (Kauffman 1987). The bracket polynomial is an invariant under the sole Reidemeister type II and III moves, and it changes when a writhe $\alpha_{+}$changes to $\alpha_{-}$. Indeed, by combining (30) and (31), we have

$$
\left\langle{ }^{\prime} \bigcirc\right\rangle=b\langle\mid\rangle, \quad\left\langle b^{\circ}\right\rangle=b^{-1}\langle\mid\rangle \quad\left(b=-a^{3}\right),
$$

that shows that the bracket polynomial is not invariant under the Reidemeister type I move (the so-called writhe move). Furthermore, we need to take care of the orientation naturally induced by the field lines. Therefore, we must include orientation and consider oriented knots $\mathcal{K}$.

Let us denote by $\operatorname{Wr}(\mathcal{K})$ the writhing number of $\mathcal{K}$, defined as usual by $\operatorname{Wr}(\mathcal{K})=\sum_{r} \epsilon_{r}$, where $\epsilon_{r}= \pm 1$, according to standard convention on crossing sites over the $r$ apparent crossings of $\mathcal{K}$. Then, let us introduce a new polynomial $V(\mathcal{K})$ defined by

$$
V(\mathcal{K})=b^{-W r(\mathcal{K})}\langle\mathcal{K}\rangle
$$

As can be easily verified, $V(\mathcal{K})$ is an isotopy invariant of the ambient space, that is,

$$
V\left(\boldsymbol{\alpha}_{+}\right)=V\left(\boldsymbol{\alpha}_{-}\right)=V\left(\boldsymbol{\alpha}_{0}\right) .
$$

The skein relations of $V(\mathcal{K})$ can be derived at once from those of the bracket polynomial. By eliminating $\left\langle L_{\infty}\right\rangle$ from the two relations (30), we have

$$
a\left\langle L_{+}\right\rangle-a^{-1}\left\langle L_{-}\right\rangle=\left(a^{2}-a^{-2}\right)\left\langle L_{0}\right\rangle .
$$

Then, by letting $\tau=a^{-4}$ and by replacing $\langle\mathcal{K}\rangle$ with $b^{W r(\mathcal{K})} V(\mathcal{K})$, we have

$$
\begin{aligned}
& V(\bigcirc)=1 \\
& \tau^{-1} V(\nearrow)-\tau V(\searrow)=\left(\tau^{\frac{1}{2}}-\tau^{-\frac{1}{2}}\right) V(\circlearrowright) .
\end{aligned}
$$

By the direct application of (36) and (37) we can see that the third skein relation of the bracket polynomial, equation (31), becomes

$$
V(\uparrow \sqcup \bigcirc)=-\left(a^{-2}+a^{2}\right) V(\uparrow)
$$

Equations (36) and (37) are the skein relations of the Jones polynomial for oriented knots. They provide a powerful tool to compute the topology of fluid knots in terms of helicity via $\tau=t^{-4 \lambda H\left(\gamma_{+}\right)}$. This completes the proof of the theorem. 


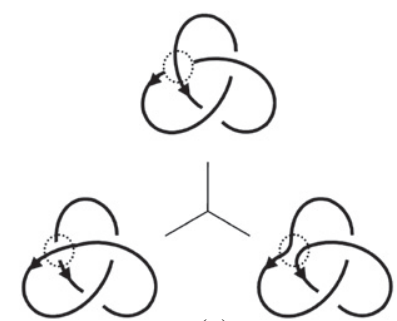

(a)

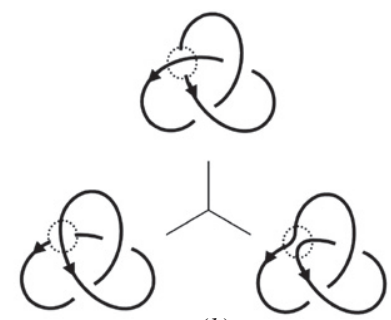

(b)

Figure 10. Reduction schemes for the left- and right-handed trefoil knots. (a) Top: left-handed trefoil knot; bottom: writhe $\boldsymbol{\gamma}_{-}$and a Hopf link $\boldsymbol{H}_{-}$, with crossing -1 . (b) Top: right-handed trefoil knot; bottom: writhe $\boldsymbol{\gamma}_{+}$and a Hopf link $\boldsymbol{H}_{+}$, with crossing +1 . The two knots are mirror images of one another.

(a)

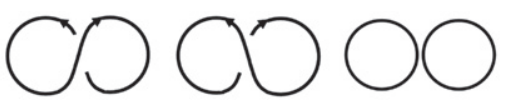

(b)

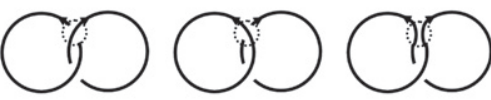

(c)

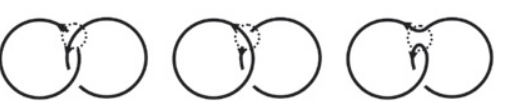

Figure 11. (a) Writhes $\boldsymbol{\gamma}_{+}, \boldsymbol{\gamma}_{-}$and disjoint union of two trivial circles $\boldsymbol{l}_{c c}$. (b) Hopf link $\boldsymbol{H}_{+}$with crossing +1 , disjoint union of circles $\boldsymbol{l}_{c c}$ and a writhe $\boldsymbol{\gamma}_{+} \cdot(c)$ Hopf link $\boldsymbol{H}_{-}$with crossing -1 , disjoint union of circles $\boldsymbol{l}_{c c}$ and writhe $\boldsymbol{\gamma}_{-}$.

\section{Jones' polynomial calculation from helicity}

In this section we provide two examples of the direct calculation of the Jones polynomial by using diagram decomposition and the skein relations (36) and (37).

\subsection{The left- and right-handed trefoil knots}

Jones' polynomial can distinguish mirror images of knots; as an example we provide explicit calculations for the left- and right-handed trefoil knots (see top diagrams of figures $10(a)$ and (b)). By re-arranging (37), we can convert a crossing in terms of its opposite plus a contribution from parallel strands, that is,

$$
V(\nearrow)=\tau^{2} V(\nearrow)+\left(\tau^{\frac{3}{2}}-\tau^{\frac{1}{2}}\right) V(\nearrow)
$$

This relation can be applied to a crossing of the trefoil knots of figure 10 (shown in the top diagrams) to decompose them in their relative contributions, given by a writhe and a Hopf link, with corresponding orientations (bottom diagrams). It is curious to note that the decomposition of the right-handed trefoil knot into a writhe $\boldsymbol{\gamma}_{+}$and a Hopf link $\boldsymbol{H}_{+}$with crossing +1 coincides with the diagram shown by Moffatt (1969, p 119) in his original paper. With reference to the left-handed trefoil of figure 10(a), we have a writhe $\boldsymbol{\gamma}_{-}$and a Hopf link $\boldsymbol{H}_{+}$. For the writhe, by using (37), we have (see figure 11(a))

$$
\tau^{-1} V\left(\boldsymbol{\gamma}_{+}\right)-\tau V\left(\boldsymbol{\gamma}_{-}\right)=\left(\tau^{\frac{1}{2}}-\tau^{-\frac{1}{2}}\right) V\left(\boldsymbol{l}_{c c}\right),
$$


that gives

$$
V\left(\boldsymbol{l}_{c c}\right)=-\tau^{-\frac{1}{2}}-\tau^{\frac{1}{2}},
$$

where $\boldsymbol{l}_{c c}$ denotes disjoint union of trivial circles. As regards to the Hopf link $\boldsymbol{H}_{+}$, we must pay attention to the relative orientation of the components. With reference to figure $11(b)$, we have

$$
\tau^{-1} V\left(\boldsymbol{H}_{+}\right)-\tau V\left(\boldsymbol{l}_{c c}\right)=\left(\tau^{\frac{1}{2}}-\tau^{-\frac{1}{2}}\right) V\left(\boldsymbol{\gamma}_{+}\right)
$$

that gives

$$
V\left(\boldsymbol{H}_{+}\right)=-\tau^{\frac{1}{2}}-\tau^{\frac{5}{2}} .
$$

Similarly for the Hopf link $\boldsymbol{H}_{-}$of the right-handed trefoil knot of figure 10(b). With reference to figure $11(c)$, we have

$$
\tau^{-1} V\left(\boldsymbol{l}_{c c}\right)-\tau V\left(\boldsymbol{H}_{-}\right)=\left(\tau^{\frac{1}{2}}-\tau^{-\frac{1}{2}}\right) V\left(\boldsymbol{\gamma}_{-}\right)
$$

that gives

$$
V\left(\boldsymbol{H}_{-}\right)=-\tau^{-\frac{1}{2}}-\tau^{-\frac{5}{2}}
$$

By comparing (43) with (45), we see that different orientations determine different polynomials.

Let us combine the results above to obtain the Jones polynomial. For the left-handed trefoil knot, say $\boldsymbol{T}^{L}$, we have

$$
\tau^{-1} V\left(\boldsymbol{\gamma}_{-}\right)-\tau V\left(\boldsymbol{T}^{L}\right)=\left(\tau^{\frac{1}{2}}-\tau^{-\frac{1}{2}}\right) V\left(\boldsymbol{H}_{-}\right),
$$

that, by using (45), gives

$$
V\left(\boldsymbol{T}^{L}\right)=\tau^{-1}+\tau^{-3}-\tau^{-4} .
$$

For the right-handed trefoil $\operatorname{knot} \boldsymbol{T}^{R}$, we have

$$
\tau^{-1} V\left(\boldsymbol{T}^{R}\right)-\tau V\left(\boldsymbol{\gamma}_{+}\right)=\left(\tau^{\frac{1}{2}}-\tau^{-\frac{1}{2}}\right) V\left(\boldsymbol{H}_{+}\right) .
$$

Thus, by using (43), we have

$$
V\left(\boldsymbol{T}^{R}\right)=\tau+\tau^{3}-\tau^{4}
$$

As we see by comparing (47) with (49), the Jones polynomial can distinguish a knot from its mirror image.

\subsection{The Whitehead link}

Let us consider the two Whitehead links with different orientations shown in the top diagrams of figure 12. With reference to the bottom diagrams of figure 12, by applying the skein relation (37) to the Whitehead link $\boldsymbol{W}_{+}$of crossing +1 , we have the relation

$$
\tau^{-1} V\left(\boldsymbol{W}_{+}\right)-\tau V\left(\boldsymbol{H}_{-}\right)=\left(\tau^{\frac{1}{2}}-\tau^{-\frac{1}{2}}\right) V\left(\boldsymbol{T}^{L}\right),
$$

and application of (37) to the Whitehead link $\boldsymbol{W}_{-}$of crossing -1 gives

$$
\tau^{-1} V\left(\boldsymbol{H}_{+}\right)-\tau V\left(\boldsymbol{W}_{-}\right)=\left(\tau^{\frac{1}{2}}-\tau^{-\frac{1}{2}}\right) V\left(\boldsymbol{F}^{8}\right),
$$

where $\boldsymbol{F}^{8}$ denotes the figure-of-eight knot shown at the bottom of figure 12(b). The latter can be further reduced according to the diagrams of figure 13. By applying (37) to the unknot with two writhes $\boldsymbol{\gamma}_{-}$(denoted by $\boldsymbol{\gamma}_{=}$), and to the Hopf link with the writhe $\boldsymbol{\gamma}_{+}$(denoted by $\boldsymbol{H}_{-}^{+}$), we have

$$
\tau^{-1} V\left(\boldsymbol{F}^{8}\right)-\tau V\left(\boldsymbol{\gamma}_{=}\right)=\left(\tau^{\frac{1}{2}}-\tau^{-\frac{1}{2}}\right) V\left(\boldsymbol{H}_{-}^{+}\right)
$$




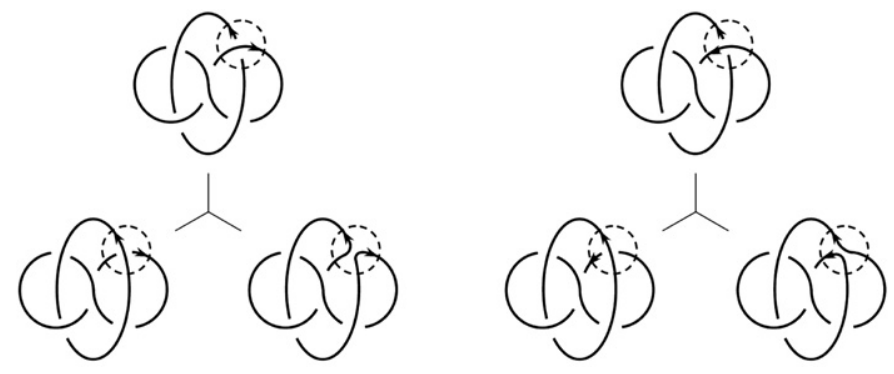

Figure 12. Reduction schemes for Whitehead links $\boldsymbol{W}_{+}$and $\boldsymbol{W}_{-}$. (a) Top: Whitehead link $\boldsymbol{W}_{+}$ with crossing +1 ; bottom: Hopf link $\boldsymbol{H}_{-}$and the left-handed trefoil knot $\boldsymbol{T}^{L}$. (b) Whitehead link $\boldsymbol{W}_{-}$with crossing -1 ; bottom: Hopf link $\boldsymbol{H}_{+}$, and a figure-of-eight $\operatorname{knot} \boldsymbol{F}^{8}$.
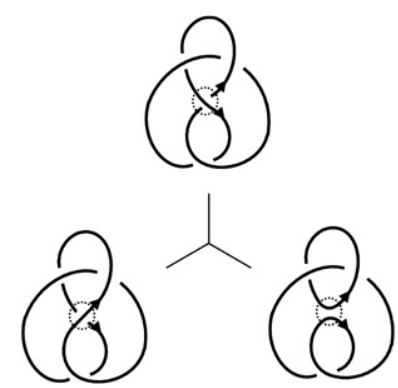

Figure 13. Top: reduction scheme for the figure-of-eight knot $\boldsymbol{F}^{8}$; bottom: the unknot with the two writhes $\boldsymbol{\gamma}_{-}$, denoted by $\boldsymbol{\gamma}_{=}$(left), and a Hopf link with a writhe $\boldsymbol{\gamma}_{+}$, denoted by $\boldsymbol{H}_{-}^{+}$.

Now, since $V\left(\boldsymbol{\gamma}_{=}\right)=1$ and $V\left(\boldsymbol{H}_{-}^{+}\right)=V\left(\boldsymbol{H}_{-}\right)=-\tau^{-\frac{1}{2}}-\tau^{-\frac{5}{2}}$, we have

$$
V\left(\boldsymbol{F}^{8}\right)=\tau^{-2}-\tau^{-1}+1-\tau+\tau^{2} .
$$

As can be easily verified, the mirror image of the figure-of-eight knot of figure 13 has the same Jones polynomial as that of equation (53). is

Hence, by substituting (45) and (47) into (50), we have the Jones polynomial for $\boldsymbol{W}_{+}$, that

$$
V\left(\boldsymbol{W}_{+}\right)=\tau^{-\frac{7}{2}}-2 \tau^{-\frac{5}{2}}+\tau^{-\frac{3}{2}}-2 \tau^{-\frac{1}{2}}+\tau^{\frac{1}{2}}-\tau^{\frac{3}{2}} .
$$

Similarly, by substituting (43) and (53) into (51), we have the Jones polynomial for $\boldsymbol{W}_{-}$, that is

$$
V\left(\boldsymbol{W}_{-}\right)=\tau^{-\frac{7}{2}}-2 \tau^{-\frac{5}{2}}+\tau^{-\frac{3}{2}}-2 \tau^{-\frac{1}{2}}+\tau^{\frac{1}{2}}-\tau^{\frac{3}{2}} .
$$

Evidently these two equations coincide, which means that different orientations of the Whitehead link have no consequences for the resulting polynomial. Thus, we can conclude that the Jones polynomial of the Whitehead link $V(\boldsymbol{W})$ is given by

$$
V(\boldsymbol{W})=\tau^{\frac{3}{2}}\left(-1+\tau^{-1}-2 \tau^{-2}+\tau^{-3}-2 \tau^{-4}+\tau^{-5}\right) .
$$

\section{Conclusions}

In this paper we have proved that the Jones polynomial of fluid knots and links under ideal conditions can be expressed in terms of kinetic or magnetic helicity. This has been 
done by re-interpreting helicity as an Abelian Chern-Simons action (section 2), following an approach similar to that used in topological quantum field theory. First we have recalled some basic concepts and techniques of knot theory (section 3) useful to introduce the $R$-polynomial for oriented knots. Then in section 4 we have proven our main result that the Jones polynomial of fluid knots and links can be determined in terms of helicity. This has been done in two steps: by using the Kauffman bracket polynomial for unoriented knots we have derived (section 4.1) the three standard skein relations, and then (section 4.2), by including orientation, we have reduced them to the skein relations of the Jones polynomial for oriented knots. In section 5, for illustration, we have computed the Jones polynomial explicitly in several cases; first, we have considered the left- and right-handed trefoil knots to show that indeed the Jones polynomial can distinguish mirror images; then we have considered the Whitehead link via the figure-of-eight knot, for which the Gauss linking number vanishes.

This work demonstrates the breath and importance of this fundamentally new topological approach to fluid dynamics based on polynomial invariants of knot theory expressed in terms of fluid helicity. This has several consequences. Conceptually, this approach provides a much richer topological context by freeing the role of helicity from its exclusive relationship with linking numbers. From an applied viewpoint, this is particularly important for all those cases, in which topology is essential, but linking numbers fail to detect it (such as for the Whitehead link). More generally, this work provides a fundamentally new role for the Jones polynomial of knots and links in terms of fluid helicity. From a foundational viewpoint, this provides further evidence of the topological character of classical field theory, and ideal fluid dynamics in particular.

In the light of modern developments in direct numerical simulations of fluid flows and advanced visiometrics, then, numerical implementation of knot polynomial calculations provides a new, powerful tool for computing topological aspects of complex fluid structures. Work in this direction, based on extracting physical information from diagram analysis by using knot theoretical concepts (Barenghi et al 2001, Ricca 2009), demonstrates this potential for future diagnostic applications.

All this will contribute to open up new directions of work, both in theory and applications. For instance, the consequences of now knowing that one can detect the unknot using the Khovanov or Heegaard Floer theories to study knots and links suggest the possibility of developing even more sophisticated tools than the knot polynomial invariants. Thus, the development of an analogous program connecting helicity to these theories might help identify some totally new topological characteristics of fluid knots. Some of our current work is in progress along these new lines of research.

\section{Acknowledgments}

XL wishes to thank Ruibin Zhang for useful discussions and advice. Financial support from the USYD Postdoctoral Fellowship program of Sydney University is also kindly acknowledged. XL and RLR wish to acknowledge the kind hospitality of the 'Ennio De Giorgi' Mathematical Research Center of the Scuola Normale Superiore in Pisa, where part of this work was carried out. We are also grateful to the anonymous referees for their valuable comments.

\section{References}

Altschuler D and Freidel L 1997 Vassiliev knot invariants and Chern-Simons perturbative theory to all orders Commun. Math. Phys. 187 261-87

Barenghi C F, Ricca R L and Samuels D C 2001 How tangled is a tangle? Physica D 157 197-206

Bar-Natan D 1995 Perturbative Chern-Simons theory J. Knot Theory Ramif. 4 503-47 
Birman J S and Lin X-S 1993 Knot polynomials and Vassiliev's invariants Invent. Math. 111 225-70

Călugăreanu G 1961 Sur les classes d'isotopie des nœuds tridimensionnels et leurs invariants Czech. Math. J. T11 588-625

Chmutov S, Duzhin S and Mostovoy J 2011 Introduction to Vassiliev Knot Invariants (Cambridge: Cambridge University Press)

Freyd P, Yetter D, Hoste J, Lickorish W B R, Millett K and Ocneanu A 1985 A new polynomial invariant of knots and links Bull. Am. Math. Soc. 12 239-46

Jones V F R 1987 Hecke algebra representations of braid groups and link polynomials Ann. Math. 126 335-88

Kauffman L H 1987 On Knots (Princeton, NJ: Princeton University Press)

Kauffman L H 1991 Knots and Physics (Singapore: World Scientific)

Labastida J M F and Pérez E 1998 Kontsevich integral for Vassiliev invariants from Chern-Simons perturbation theory in the light-cone gauge J. Math. Phys. 39 5183-98

Le T T Q, Murakami J and Ohtsuki T 1998 On the universal perturbative invariant of 3-manifolds Topology 37 539-74

Maxwell J C 1873 A Treatise on Electricity and Magnetism (Oxford: Clarendon)

Moffatt H K 1969 The degree of knottedness of tangled vortex lines J. Fluid Mech. 35 117-29

Moffatt H K and Ricca R L 1992 Helicity and the Călugăreanu invariant Proc. R. Soc. A 439 411-29

Peskin M E and Schoeder D V 1995 An Introduction to Quantum Field Theory (Reading, MA: Addison-Wesley)

Przytycki J H and Traczyk P 1987 Conwav algebras and skein equivalence of links Proc. Am. Math. Soc. 100 744-8

Ricca R L 2009 Structural complexity and dynamical systems Lectures on Topological Fluid Mechanics (SpringerCIME Lecture Notes in Mathematics vol 1973) ed R L Ricca (Berlin: Springer) pp 169-88

Ricca R L and Moffatt H K 1992 The helicity of a knotted vortex filament Topological Aspects of the Dynamics of Fluids and Plasmas (NATO ASI Series E: Applied Sciences vol 218) ed H K Moffatt et al (Dordrecht: Kluwer) pp 225-36

Ricca R L and Nipoti B 2011 Gauss linking number revisited J. Knot Theory Ramif. 20 1325-43

White J H 1969 Self-linking and the Gauss integral in higher dimensions Am. J. Math. 91 693-728

Whitehead J H C 1947 An expression of Hopf's invariant as an integral Proc. Natl Acad. Sci. USA 33 117-23

Witten E 1989 Quantum field theory and the Jones polynomial Commun. Math. Phys. 121 351-99

Woltjer L 1958 A theorem on force-free magnetic fields Proc. Natl Acad. Sci. USA $44489-91$ 